\title{
Development of Electric Vehicle Simulator for Performance Analysis
}

\author{
ChanHo Park, MinHo Kwon, Nak-Tak Jeong, Seulgi Lee, Myung-Won Suh, \\ Hyun-Soo Kim, Sung-Ho Hwang* \\ Department of Mechanical Engineering, Sungkyunkwan University,,Seoburo, Suwon, Korea \\ *Corresponding Author: hsh@me.skku.ac.kr
}

Copyright (C) 2014 Horizon Research Publishing All rights reserved.

\begin{abstract}
The goal of this paper is to invent a simulation tool for developers of electric vehicle unit system. An electric vehicle simulator structured by MATLAB/SIMULINK can change all kinds of important parameters to use GUI and observe the results. Also, if it has a new MATLAB/SIMULINK model or an experimental data of an invented component, it can be applied to perform a simulation. Additionally, we conducted a simulation in a virtual environment with a model, inventing practical driver conduct, and thus we could obtain a practically reliable simulation data. On this paper, we present each characteristic briefly and finally set Nissan Leaf vehicle as a simulator to show possibilities of a simulator as the simulator obtains similar results compared with a practical vehicle.
\end{abstract}

Keywords Virtual Integrated Development Environment, Electric Vehicle, Electric Vehicle Simulator, Component Design

\section{Introduction}

Because of increasing fuel efficiency restrictions over recent years, the Electric-Vehicle market is growing. Also, global vehicle makers are following this trend. But when the vehicle parts are changed, anticipating the performance is very difficult. So this research's goal is to develop an environment that reflects the vehicle's powertrain for design purposes. For this reason, we use MATLAB/Simulink to make a major components library, and build an electric vehicle model by using the made library. This model is verified from real car data. Also we use the $3 \mathrm{D}$ rendering tool Vega-prime to make a result that approximates the facts with a real driver model and various traffic conditions. So we can expect to utilize this developed simulation tool to make electric vehicle parts.

\section{Development of theelectric vehicle models by using MATLAB/Simulink}

The first step to set an electric vehicle simulator is to structure properly fast and easily adoptable model for noticing changes of vehicles. We have set up a model which presents a power flow of practical vehicle to adopt changes of vehicles easily.[1] Moreover, if a vehicle was only assembled by using formulas, already structured parts of vehicles almost cannot be reused when a part of vehicle is changed. For resolution, most of models have been structured by using Map-data through experiments. Besidesit, the main concern is basic vehicle characteristics such as a vehicle SOC change, a maximum speed, and a gradeability. If an inverter and a motor model applied complicated calculations is formed in a numerical approach, a simulation time will be ten timeslonger than real time with intel core 2 duo level equipment. For the original purpose to compose real-time virtual environment, we describe simple efficiency model and map data of torque-speed instead equations to reduce simulation time.[2]

\subsection{The Electric Vehicle Components Library}

SimDriveline' is a library offered by MATLAB/Simulink to analyze the behavior characteristics. Using this library makes it easy to model; for example, the following figure is a motor model that was made by 'SimDriveline' and Map data in this simulator.

The motor model was composed of map data. Basically, we use map data regarding the maximum torque at various input speeds. Block A calculates the maximum torque from the input speed. Block B uses efficiency map data for getting the motor efficiency. Block $\mathrm{C}$ calculates the electrical output power. Through the library composed above, using the map data provides accurate results.

\subsection{Composition of Electric Vehicle by Using Developed Library}

In the case of an electric vehicle, it is simpler than other eco-friendly concept cars. The electric vehicle was composed of a battery, inverter and motor. The Nissan Leaf can be built by using the developed library, as in Figure 2. 
In Figure 2, there is a driver model for effective simulation of various driving cycles, and which the controller can control. In this model, there is only the final reduction gear, without transmission. But this research supports not only the final reduction gear, but also A/T gear, so a user can easily add a gear set to the vehicle model.

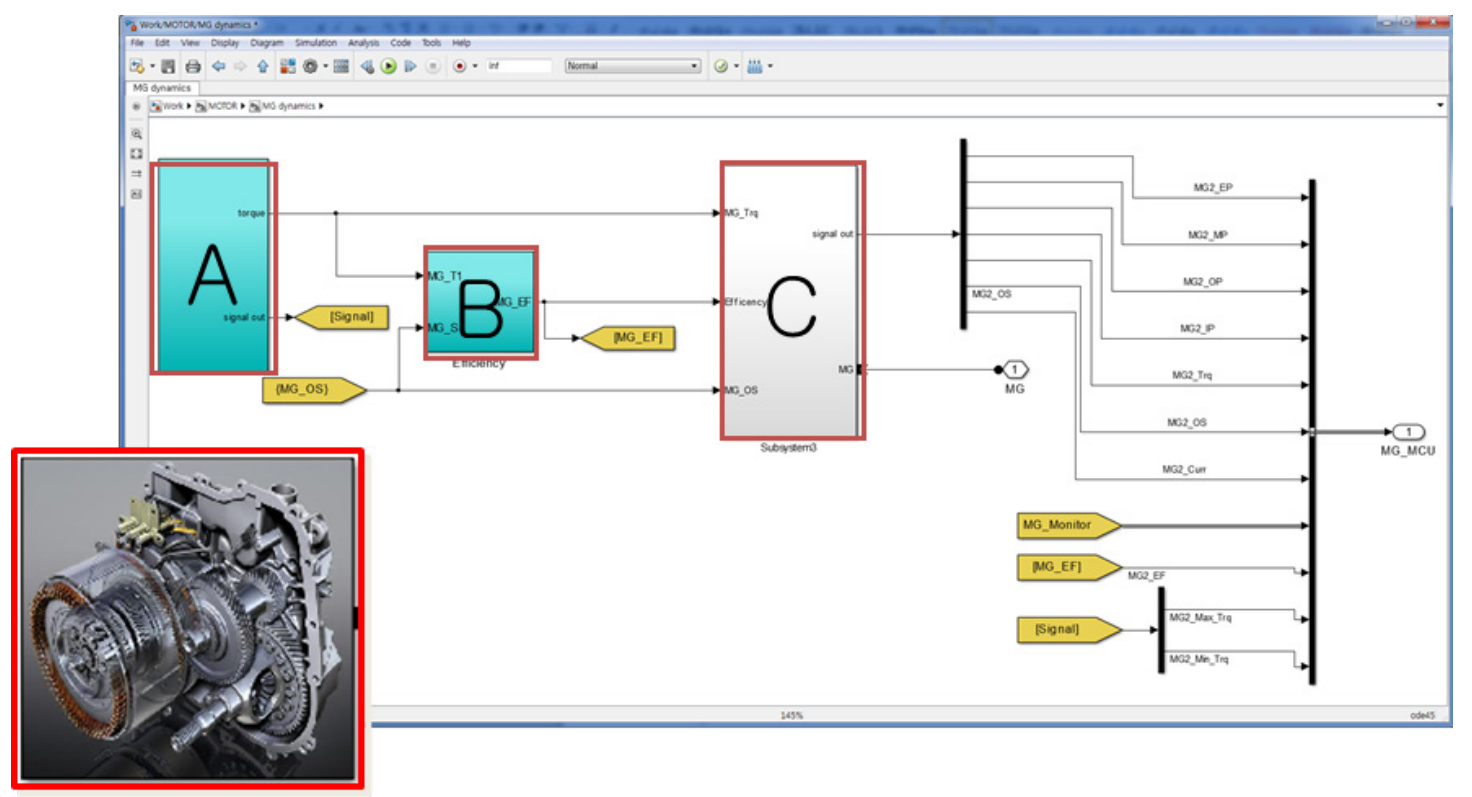

Figure 1. Motor library

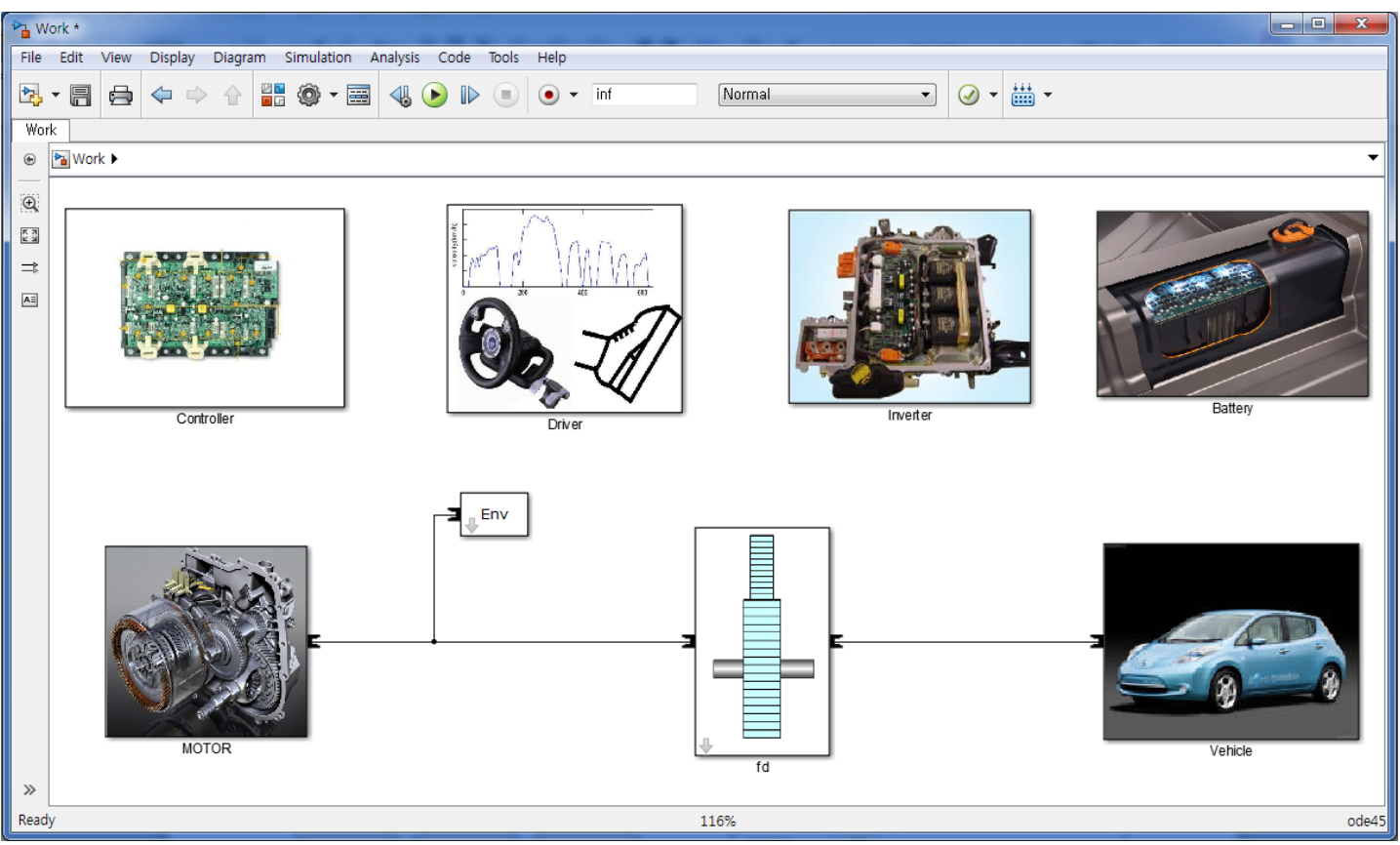

Figure2. Composition of Electric Vehicle 


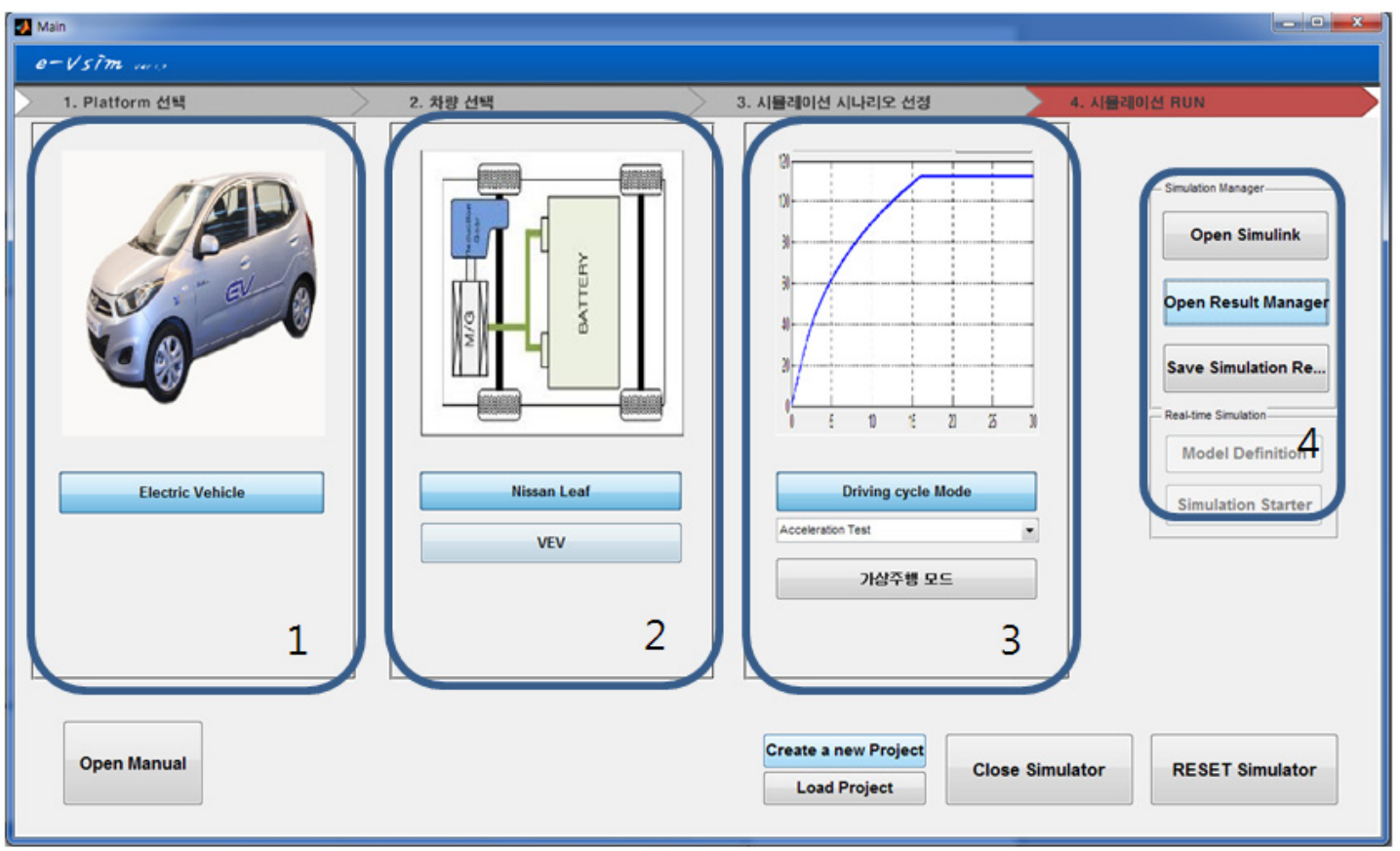

Figure3. GUI for Making Simulation Scenario

\section{Development of Virtual Environment}

Even if a well-made model was given, an ordinary user would not make the best use of a given model. Thus we have intended to supply GUI for comfortable use. Furthermore, the difference between this model andexisting few simulation tools is making the virtual environment for actual drivers to drive the model, and thus it makes possible to evaluate performance of vehicle as real driving. If an accuracy of a model is confirmed, this simulator can be used for research and development in various conditions.

\subsection{GUI for Convenience of Users}

This research's goal is to develop the environment for an electric vehicle. In other words, our goal is to develop a simulation tool. So, this simulator is made by MATLAB/Simulink, but nevertheless, people who are not experienced with MATLAB/Simulink can use this tool very easily. To achieve this goal, the simulation tool offers a GUI for user convenience.

In the above figure, our simulation tool's GUI is separated into four parts. The first part is choosing the platform of vehicle, and hence a previously given hybrid vehicle[3], an electric vehicle, and PHEV vehicle can be selected. The second part is selecting the basic model in each platform, such as Volt or Leaf. The third part is selecting the various driving cycles or tests, such as FTP 72, EDUC, JAPAN 10_15, acceleration test, or range test. Additionally there are some buttons to connect to a real time driving simulator. The fourth part is for calling the Simulink, or checking the result. 


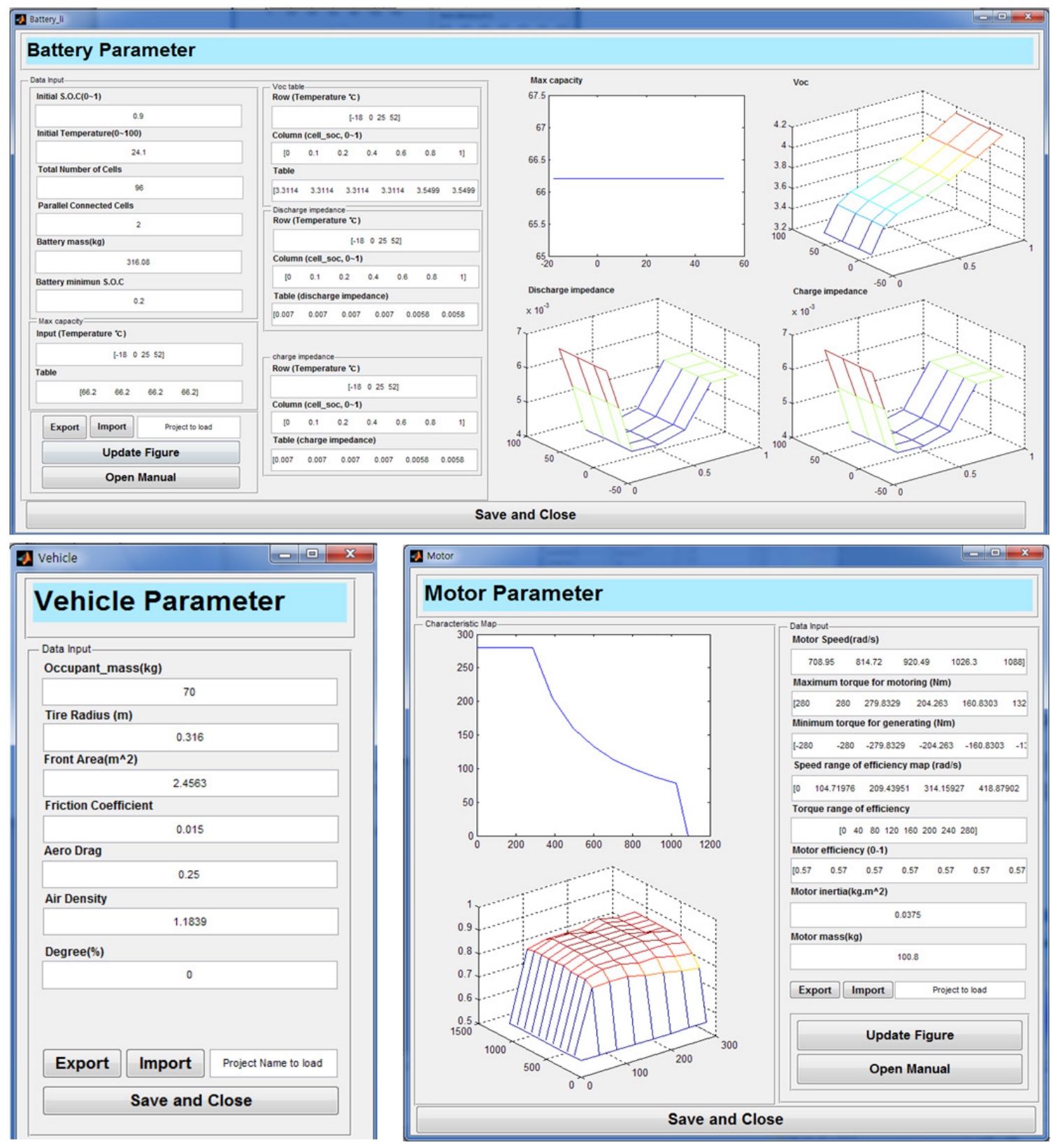

Figure4. GUI for Changing Parameters

This simulator uses a lot of map data to make the library. As in Figure 4, the user can easily change the map data and parameters on the GUI.

\subsection{Build of the Driving Simulator}

The most distinguishing feature is the driving simulator. The user can drive their own model in real time. This result reflects the traffic conditions, so the result is more realistic. The user uses the GUI to compile and load the MATLAB/Simulink model to AD5435, which is the real time simulator, and is based on Linux. Under the figure is a photo of the driving simulator.

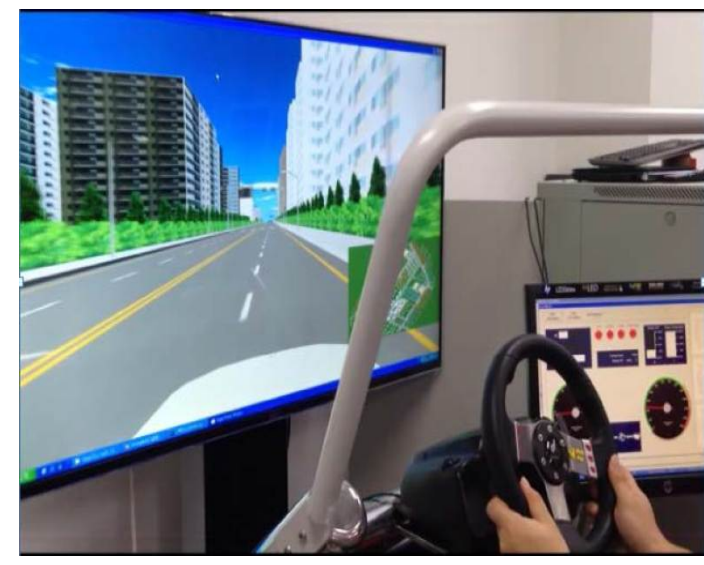

Figure5. Virtual driving with Developed Model 
Table 1. The parameters[4] and simulation result of the Nissan Leaf

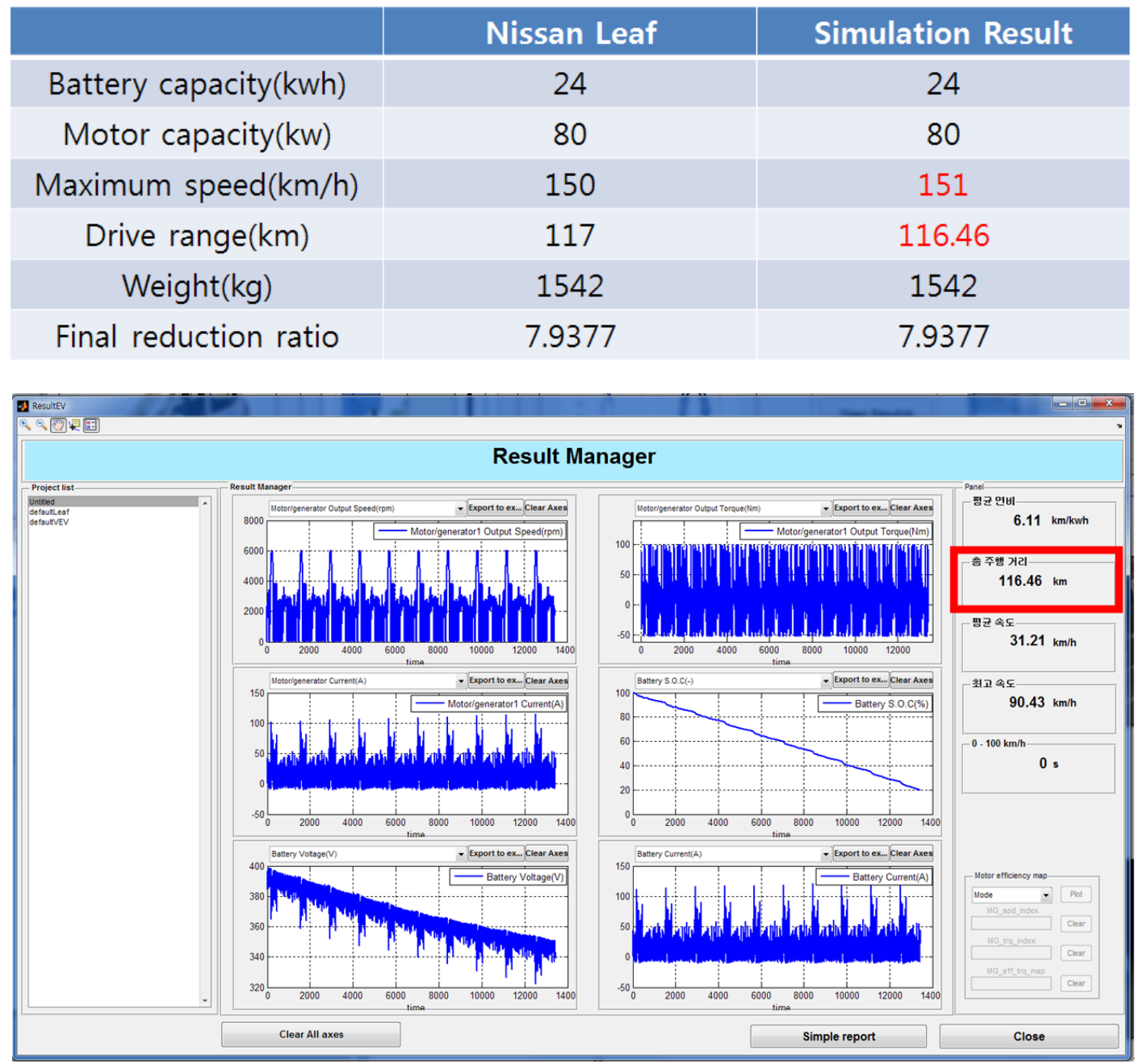

Figure6. The Result of EV Range Test

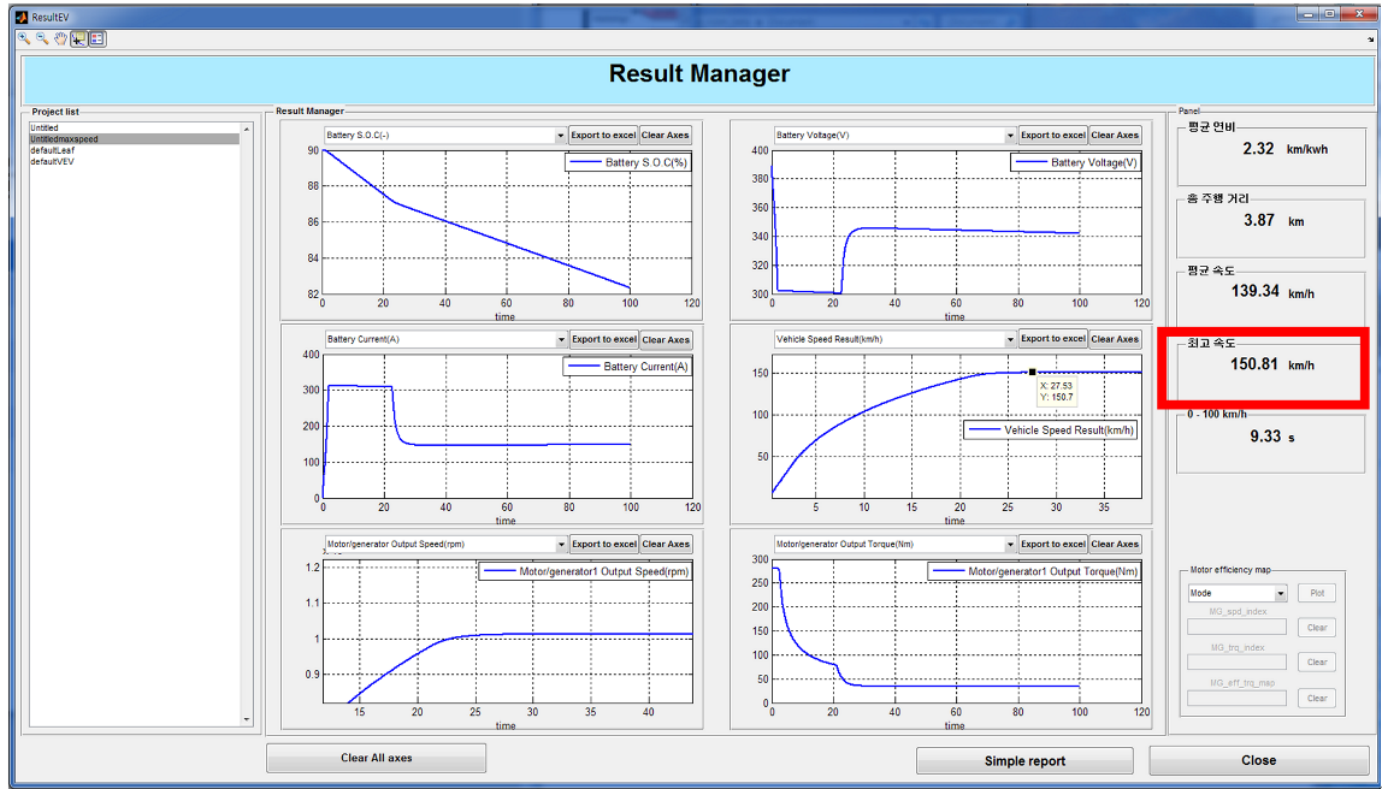

Figure7. The result of Acceleration Test 


\section{Simulation Results}

In this research, people who are not familiar with MATLAB/Simulink can easily make their own model, and check the result, by using the GUI at every stage.

Each graph can compare various data, and can export data, too. To compare the simulation result and real car data, the Nissan Leaf is built, using the obtained parameters and the made library. Figure 6 is a Drive range test, and Figure 7 is an acceleration test. Table 1 compares the results, and shows it has over $99.3 \%$ accuracy.

\section{Conclusions}

We developed a virtual environment for simulating electric vehicle performance. So we built an electric vehicle components library in MATLAB/Simulink. Also, the simulator provides some Vehicle models that are composed using the developed library. A user can easily use the simulator tool using the GUI, so people who have never used MATLAB/Simulink can develop the componentry of electric vehicles. This simulation tool is expected to shorten the development time.

\section{Acknowledgements}

This research was supported by Development of VIDE for development strategy and vehicle system design for future green car(No.10035209) funded the Korea government Ministry of Trade, Industry and Energy.

\section{REFERENCES}

[1] Karen L. Butler, MehrdadEhsani, PreyasKamath (1999, November). A Matlab-Based Modeling and Simulation Package for Electric and Hybrid Electric Vehicle Design, IEEE TRANSACTIONS ON VEHICULAR TECHNOLOGY, VOL. 48, NO. 6.

[2] Ryan C. Kroeze, Philip T. Krein (2008). Electrical Battery Model for Use in Dynamic Electric Vehicle Simulations, IEEE Power Electronics Specialists Conference (PESC).

[3] Jinhyun Park, Choong-Min Jeong, ChaOh Ma, Myung-Won Suh, Hyun-Soo Kim and Sung-Ho Hwang (2012, April). Development of Real-time Simulation Environment for Performance Analysis of Eco-friendly Vehicle, Trans. Korean Soc. Mech. Eng. A, Dynamics and control field, 47-53 (7 pages).

[4] John G. Hayes, R. Pedro R. de Oliveira, Sean Vaughan, Michael G. Egan (2011). Simplified Electric Vehicle Power Train Models and Range Estimation, IEEE Vehicle Power and Propulsion Conference (VPPC) 